González, G. \& Becerril, R. (2013). El recorrido investigador de un educador novel explicado desde una perspectiva autobiográfica. Revista Electrónica Interuniversitaria de Formación del Profesorado, 16 (3), 61-78.

DOI: http://dx.doi.org/10.6018/reifop.16.3.186511

\title{
El recorrido investigador de un educador novel explicado desde una perspectiva autobiográfica
}

\author{
Gustavo González Calvo, Raquel Becerril González \\ Universidad de Valladolid
}

\section{Resumen}

Nuestro artículo se centra en el camino investigador seguido por un maestro especialista en Educación Física desde su etapa de formación inicial hasta la inserción profesional en diferentes niveles educativos. El objetivo es reflejar la manera en que los relatos autobiográficos (en forma de autonarrativas y diarios de clase) permiten llevar a cabo una investigación sistemática de la propia práctica, favoreciendo la comprensión, transformación y enriquecimiento de la enseñanza de manera crítica y razonada. La conclusión a la que llegamos es clara: la práctica reflexiva e investigadora llevada a cabo por el docente permite no sólo cuestionar y modificar su perspectiva acerca de la enseñanza, sino también debatir y evitar ciertas concepciones sobre la profesión que ha elegido y las obligaciones que ésta implica.

\section{Palabras clave}

Diario de clase; Historia de vida; Identidad profesional; Formación inicial y permanente del profesorado.

\section{Autobiographical research track of a novice teacher}

\section{Abstract}


This article focuses on the research track followed by a PE teacher from the initial stage of his formation and throughout his professional experience in different educational levels. It shows the way in which autobiographical accounts (in the shape of narratives and class diaries) favour a systematic study of one's own practice, enhancing its understanding, transformation and improvement from both a reasoned and critical perspective. The conclusion is evident: reflective practice gives not only the possibility of calling into question and modifying one's attitude towards teaching, but also of debating and avoiding certain assumptions about the job chosen and the obligations derived from it.

\section{Key words}

Class diary; Life history; Professional identity; Initial and continuous formation of teachers.

\section{Introducción}

Cuando nos planteamos formar parte de este monográfico, nos asaltó la siguiente duda: ¿qué podemos aportar, a la comunidad científica y educativa, sobre nuestra breve experiencia como investigadores?

Como educadores (e investigadores) noveles que somos, teníamos claro que nuestro objetivo no podría ser "dar lecciones" sobre qué tipo de paradigma investigador puede resultar más apropiado, ni sobre la metodología a adoptar en función del tipo de investigación que se quiera desarrollar, ni tan siquiera dar a conocer de forma profunda algunas herramientas metodológicas cada vez más presentes en el mundo de la investigación educativa.

En ese momento, creímos que lo más conveniente sería intentar arrojar un poco de luz que permitiera al lector comprender las inquietudes, percepciones, pensamientos, miedos y expectativas que se han ido abriendo paso, en el mundo de la investigación, en un docente novel. De esta manera, el protagonista del artículo es un maestro especialista de Educación Física que se puede considerar inexperto (lleva cinco años en la profesión). Asimismo, ha impartido docencia en la etapa de Enseñanza Secundaria y como profesor asociado en la universidad.

Su periodo de formación inicial resultó ser determinante en dos aspectos. En primer lugar, fue durante esta etapa en la que se despertó un especial interés por el ámbito de la investigación y, más concretamente, por la investigación educativa. La concesión de una beca de colaboración con la Universidad de Valladolid, en el año 2005, llevaba implícita una aproximación a los métodos cualitativos y al empleo de las narraciones biográficas como modo de reflexión en la profesión docente.

En segundo lugar, y tras diferentes inmersiones en otros ámbitos de la investigación, surge la profunda convicción de que, contrariamente a lo que pueda pensarse (e, incluso, en contra de sus planteamientos iniciales), en el ámbito de la educación también se puede (y se debe) investigar. Es, quizá, este segundo aspecto, el que condiciona su reivindicación de otorgar un mayor estatus a la profesión de educador físico.

En definitiva, pretendemos mostrar cómo los relatos autobiográficos pueden erigirse en una herramienta de reflexión e introspección gracias a la que el docente es capaz de enfrentarse a su propia vida, experiencias y vivencias en una suerte de "viaje al pasado". Estas vivencias cobran, para el educador, un significado diferente de cómo las vivió entonces, y sirven para dar sentido a sus búsquedas y decisiones actuales. Más aún, la vida 
del educador no puede comprenderse si no va ligada a la vida del alumnado, de manera que conocerse mejor como persona y profesional ayuda a ser mejor educador y, en consecuencia, mejorar la vida y el aprendizaje de los escolares (González \& Barba, en prensa).

Nos mostramos convencidos de que, compartir aquí un proceso de investigación de estas características, puede ayudar a otros docentes (o futuros docentes) a emplear las narrativas como instrumento que permite transformar y enriquecer su práctica pedagógica. Se trata de un instrumento de transformación social que permite dar "voz" (y palabra) a un colectivo que, actualmente, raramente es escuchado. Por último, si estas historias son compartidas con otros educadores, estaremos en condiciones de tejer un entramado de confianza, entendimiento e igualdad por medio del (re)encuentro con las historias de los demás. Gracias a ello, construiremos un conocimiento crítico sobre la escuela y, al tiempo, una forma de asesoramiento, formación y apoyo para el profesorado (Martínez, 2008) por medio de una actividad formadora común.

\section{Marco teórico}

En la redacción de este escrito nos apoyamos en dos métodos básicos que ayuden a vislumbrar que otro tipo de investigación, en educación, es posible: a) la producción de relatos de corte autobiográfico como método de investigación en educación; y b) la (auto)etnografía.

\section{La producción de relatos de corte autobiográfico como método de investigación en educación}

Los relatos de corte autobiográfico, como estrategia de reflexión y toma de conciencia de las creencias implícitas del docente, ha ido cobrando mayor importancia en el campo de las Ciencias Sociales a finales del siglo pasado (Delgado \& Gutiérrez, 1999), constituyéndose hoy día en un planteamiento primordial en la investigación educativa. Trabajar con esta herramienta es un ejercicio indispensable y conveniente para el conjunto de investigadores del ámbito educativo y para el conjunto de la comunidad científica española, que está comenzando a producir una cantidad admirable de estudios cualitativos si se tiene en cuenta los pocos años de tradición investigadora que lleva acumulada (Silvennoinen, 1994; Devís y Sparkes, 2001; Sparkes y Smith, 2001; Brown, 2003; Pascual, 2003; Sparkes, 2003; Barbero, 2006; Sparkes y Devís, 2007; Martínez, 2005; Pulido et al., 2009). En buena parte, se debe a que este tipo de relato "es un marco adecuado para comprender el pensamiento y la práctica de los profesores” (López, 2002, p. 10).

Se erige, asimismo, en un proceso intencional y sistemático que permite rememorar y reflexionar sobre lo que uno piensa, siente y el modo en que actúa (Bolívar, Domingo, \& Fernández, 1998, 2001; Devís \& Sparkes, 2004; Silvennoinen, 2001; Sparkes, 1994, 2004). En este sentido, el relato (auto)biográfico permite, entre otras cosas, "poner a prueba y comprender las experiencias que el docente ha vivido y extraer los aspectos que considera más relevantes" (González \& Martínez, 2009, p. 35).

En el marco del artículo que aquí presentamos, entendemos que emplear este tipo de relatos en el ámbito de la investigación educativa permite reconocer la razón de ser de determinadas situaciones mediante la descripción que de ellas hace su protagonista lo que, a su vez, requiere hacer uso de la práctica reflexiva, ya que es necesario recordar con un orden lógico las vivencias pasadas que han marcado el desarrollo e interpretarlas en función de las características particulares del presente. Es decir, permiten acercarnos a la comprensión del pensamiento y práctica del educador. 


\section{La (auto)etnografía como método de investigación en educación}

Desde una aproximación general, la etnografía es un método de investigación social en el que se lleva a cabo una descripción o reconstrucción analítica de escenarios y grupos culturales (Goetz \& LeCompte, 1988). Es decir, es una manera de estudiar y describir la cultura humana, entendiendo por cultura "todo aquello que ha sido aprendido o producido por un grupo de personas en un contexto determinado" (Buendía, 2010, p. 26).

Su principal característica es que el investigador participa, abiertamente o de manera encubierta, en la cotidianeidad de las personas durante un periodo de tiempo "observando qué sucede, escuchando qué se dice, haciendo preguntas; de hecho, haciendo acopio de cualquier dato disponible que pueda arrojar un poco de luz sobre el tema en que se centra la investigación" (Hammersley \& Atkinson, 1994, p. 15).

Dentro del contexto que aquí interesa, la etnografía educativa se puede definir como la aportación de "valiosos datos descriptivos de los contextos, actividades y creencias de los participantes en los escenarios educativos" (Goetz \& LeCompte, 1988, p. 41).

Para Velasco y Díaz (2004, p. 10), la etnografía educativa "no es más que el resultado de aplicar una práctica etnográfica y una reflexión antropológica al estudio de la institución escolar". Esta perspectiva implica llevar a cabo una práctica reflexiva personal en el contexto escolar en que se desarrolla la profesión.

La etnografía, en el ámbito educativo, aparece como alternativa a los enfoques de investigación de corte positivista. En palabras de Fernández:

"Lo que persigue la observación etnográfica es poner la visión e interpretación de los procesos vivos donde antes sólo había un recuento de entradas y salidas, una especie de contabilidad de lo que entra y sale de la escuela. Con ello no pretendemos negar el pan y la sal a los estudios demográficos, las encuestas, etc. [... ]. Pretendemos, tan sólo, romper una lanza, o lo que sea, a favor de la investigación cualitativa, que tiene sus propios métodos y sus propios resultados, siempre intransferibles" (Fernández, 1985, p. 87).

Esta alternativa a la metodología positivista ofrecida por la etnografía educativa es reconocida como muy útil y valiosa para el educador (Álvarez, 2008; Barba, 2001; Fernández, 1985; Goetz \& LeCompte, 1988; González, 2013; González et al., 2013; Pallma \& Sinisi, 2004; Rockwell, 2009; Woods, 1998) ya que, como explica San Fabián (1992, en Álvarez, 2008, p. 1), "al ser la educación un proceso cultural por el que niños y jóvenes aprenden a actuar adecuadamente como miembros de una sociedad, hace de ella un ámbito particularmente idóneo para la investigación etnográfica". De esta manera, el investigador del campo de la educación tiene a su disposición una excelente alternativa para la "descripción, interpretación y explicación del universo social, así como de los fenómenos educativos que tienen lugar en él" (Goetz \& LeCompte, 1988, p. 54).

Las características más importantes de la etnografía escolar, de acuerdo con Álvarez (2008), son:

1. La participación prolongada en el contexto a estudiar para dar cuenta del punto de vista de los "nativos": se refiere a la necesidad de convivir con el grupo a estudiar durante periodos de tiempo continuados para comprender las interacciones que se producen entre sus miembros y los significados que se construyen.

2. La observación participante del investigador como "nativo marginal": hace referencia al hecho de que el etnógrafo nunca es uno más en el grupo que estudia, 
pero debe intentar integrarse lo máximo posible para realizar adecuadamente su investigación.

3. La descripción reflexiva con carácter holista: supone, para el etnógrafo, la necesidad de realizar un trabajo en el que se relacione a los individuos, grupos u organizaciones con su entorno social, económico, físico y simbólico de manera global.

Las particulares características de nuestro relato, en el que investigador e investigado son la misma persona, llevan a que la observación participante no se haya realizado guardando cierta distancia con aquello que se observaba. Por esta razón, quizá, debamos hablar de autoetnografía más que de etnografía. Ambas comparten las mismas características anteriormente descritas, con la salvedad de que, en la autoetnografía, la frontera entre lo social y lo personal se diluye (Feliu, 2007). En este sentido, Ellis y Bochner (2000, en Sparkes, 2004, p. 54) consideran que "la autoetnografía es un género autobiográfico de escritura e investigación que despliega múltiples capas de consciencia, conectando lo personal con lo cultural".

Dada la posibilidad de que nuestro artículo pueda servir como referente a otros educadores que se enfrentan a sus primeros años de profesión, la autoetnografía resulta útil en dos sentidos. El primero de ellos es que, con este modelo de escritura, los autores permiten:

"tomar parte en una experiencia que puede revelarnos no sólo cómo fue para los autores, sino cómo podría ser, o cómo fue para nosotros como lectores" (Sparkes, 2004, p. 56). El segundo se justifica en la medida en que "la narración del viaje permite la comprensión del proceso narrado, [...], no se trata de resolver un misterio, sino de dar claves para la empatía, y por lo tanto pasar de la superioridad del autor a la igualdad con el lector" (Feliu, 2007, p. 268).

\section{Objetivos}

Los objetivos de la investigación se circunscriben a los siguientes aspectos:

a. Reflejar el empleo, por parte del docente, de algunas herramientas básicas para investigar su propia práctica.

b. Despertar una cierta (pre)disposición en los educadores inexpertos a investigar su propia práctica como modo de mejorar en su profesión.

c. Trazar un pequeño recorrido acerca del modo en que van evolucionando la identidad profesional y las inquietudes investigadoras del docente novel.

En el presente apartado se mostrará el proceso de Espacio Mestizo con evidencias de los cambios sucedidos.

\section{Consideraciones metodológicas}

La recogida de la información que sustenta nuestro escrito surge durante el curso escolar 2005/2006, en la etapa de formación inicial como maestro especialista de Educación Física del protagonista.

Los registros que van apareciendo contienen opiniones, reflexiones individuales y compartidas con otros profesionales, experiencias, vivencias y, en definitiva, las inquietudes del maestro inexperto, adoptando todos ellos el formato de diarios de clase. 
En este sentido, hemos de destacar el convencimiento temprano del docente acerca del potencial formativo que tienen los registros de aula y los relatos autobiográficos. Se entiende, así, que la reflexión y el análisis de la información son procesos que han ido siempre de la mano al desarrollo de la identidad profesional del educador; esto es, no es algo que se haya llevado a cabo una vez concluido el proceso investigador.

A partir del curso escolar 2009/2010, y ejerciendo como maestro interino, nuestro protagonista decide continuar su rutina de reflexión a partir de los relatos personales. De esta manera, y tras ir ejerciendo la enseñanza en diferentes centros y niveles educativos (desde la etapa de Educación Primaria, pasando por la Enseñanza Secundaria hasta desempeñar el cargo como profesor asociado universitario), a la finalidad formativa de estos escritos se le añade la investigación propia de los estudios de doctorado. En este sentido, se ha contado con la ayuda de otros profesionales de la enseñanza y profesores universitarios de diferentes áreas de conocimiento interesados igualmente en la formación permanente a través de la producción y análisis de relatos autobiográficos.

Por lo tanto, contamos con un largo proceso de producción y registro de relatos personales que emanan del convencimiento de su función (auto)formativa, un proceso en el que las reflexiones se hacen cada vez más sistemáticas, en el que recolección y análisis van de la mano y progresan juntos (Taylor \& Bogdan, 1998, p. 158).

Hemos de señalar, asimismo, que durante la última etapa como profesor novel, el instrumento más utilizado para la producción y extracción de relatos autobiográficos han sido los diarios de clase. Éstos, como explica Zabalza (2008, p. 15), "son los documentos en los que profesores y profesoras recogen sus impresiones sobre lo que está sucediendo en sus clases". Son también un instrumento de registro de experiencias educativas con la intención de ser posteriormente analizadas y compartidas (Smyth, 1991).

A este respecto, distintos autores (Chacón \& Chacón, 2006; Liston \& Zeichner, 2003; Surbeck, Han, \& Moyer, 1991; Zabalza, 2008; Zeichner \& Liston, 1999) resaltan que los diarios constituyen una herramienta motivadora de procesos críticos y reflexivos, que ayudan a ampliar el pensamiento acerca de los acontecimientos que tienen lugar en el aula y que posibilitan que los docentes profundicen en sus propias acciones e ideas sobre los procesos de enseñanza/aprendizaje.

El diario tiene dos perspectivas: una sincrónica y puntual, ya que se centra en la narración de lo sucedido; y otra diacrónica, por cuanto presenta la evolución de los hechos que se narran, lo que permite observar el cambio de opiniones, impresiones, problemas y actuaciones del docente a lo largo del tiempo (Zabalza, 2008).

Gracias a esta herramienta, el docente puede enfrentarse todos los días, con rigor y con método, a los hechos, a preguntarse por ellos, a analizarlos detenidamente y a ver a dónde nos llevan. En este sentido, ser consciente sobre el papel de las experiencias vividas ayuda a que éstas se objetiven, haciendo que la maraña de acontecimientos vividos durante el día cobren orden, impidiendo que se diluyan en la memoria (Latorre, 1992, p. 231).

\section{Reconstrucción biográfica a partir de relatos: el camino investigador de un docente novel}

El análisis de la historia de vida y los diarios de clase del educador se han analizado partiendo, previamente, del establecimiento de una serie de criterios que nos permitiera agruparlos en diferentes categorías, siendo éstas:

1. Los recuerdos sobre el pasado como estudiante universitario de Educación Física. 
2. La evolución del yo docente.

3. Las motivaciones profesionales y cómo se vinculan con la labor investigadora del docente.

4. El modo en que van evolucionando las inquietudes investigadoras del educador.

\section{Las primeras experiencias con la investigación educativa: las narrativas autobiográficas}

El primer acercamiento del docente al mundo de las autonarrativas viene de la mano de uno de sus profesores universitarios, gracias a haber cursado una asignatura dedicada a la investigación cualitativa dentro de su formación inicial como maestro.

Aproximarse a la literatura existente sobre este tipo de metodología le sirvió para irse abriendo camino al descubrimiento de que las historias de vida son un excelente recurso para comprender por qué uno es, actúa y siente de una determinada manera (y no de otra). Algunas de sus lecturas fueron determinantes en la concepción que se iba configurando sobre la investigación cualitativa (entre sus referentes destacan, por ejemplo, Devís \& Sparkes, 2004; Devís \& Sparkes, 1999; Silvennoinen, 1994; Silvennoinen, 2001; Sparkes, 1994, 2001, 2004).

Un año más tarde, y gracias a una beca de colaboración (de las que hoy día tanto escasean $y$, al mismo, tan necesarias) con la Universidad de Valladolid, el educador pudo profundizar más en este instrumento de investigación:

"Participar en la recogida de datos, procesado y categorización de relatos de vida, dentro de una investigación que tenía como objetivo principal conocer cómo afectaban al desarrollo del plan de estudios de la diplomatura de Educación Física las diferentes trayectorias vitales de los alumnos que decidieron optar por esta titulación, me permitió comprender más acerca de la investigación cualitativa" (Historia de vida).

Ya más experimentado con esta estrategia, el docente se aventuró a escribir su propio relato de vida en lo referente a cómo una lesión tuvo una influencia determinante en la ruptura de sus proyectos de vida (hasta entonces dominantes) y su repercusión en el modo en que orienta y concibe la enseñanza de la Educación Física. Esta propuesta vio la luz en forma de publicación (véase González \& Martínez, 2009):

"Desde una perspectiva profesional la redacción de este escrito supuso una herramienta útil para reflexionar, profundizar en mis pensamientos, recapacitar sobre los aspectos éticos de este tipo de metodologías y entender, en definitiva, que la elaboración de una historia de vida afecta directamente a aquel que la escribe, lo que explica la dificultad para hacer públicas cuestiones que se consideran íntimas. Desde una perspectiva personal, reconozco que fue complicado escribirlo, despertándose en mí en muchos momentos mi lado más melancólico" (Historia de vida).

A raíz de la decisión del educador de emprender una tesis doctoral empleando una metodología cualitativa, tuvo que redactar de nuevo su historia de vida, ahora más extensa en cuanto a volumen de recuerdos y vivencias para, así, poder ahondar en determinados pensamientos. Paradójicamente, a partir de la nueva escritura, se diluye ese resquicio taciturno para servir de mejora en su estado de ánimo:

"Ser el investigador y el sujeto investigado al mismo tiempo ha hecho que la redacción de la historia de vida se haya convertido, por momentos, en una especie de "psicoterapia", en algo así como una "vía de escape", pues he comprobado que, aunque a veces es duro, dedicar un tiempo a escribir acerca de mis experiencias pasadas ayuda a sentirme mejor conmigo mismo" (Historia de vida). 
Se constata así que la construcción de la identidad profesional se va realizando a través del conocimiento y la indagación que al docente le entrega su experiencia, siendo los referentes pedagógicos recibidos como universitario los que han impulsado y estimulado la capacidad reflexivo-investigadora de nuestro educador.

Las primeras experiencias con los diarios de clase: de maestro a investigador de la propia práctica

La primera experiencia con la utilización del diario de clases surge gracias a la asignatura del Prácticum, obligatoria dentro del plan de estudios de maestro de Educación Física. Este primer acercamiento con la realidad de un centro escolar implicaba, para nuestro educador, poder dejar constancia de su práctica docente y de la responsabilidad que ésta implica:

"El propósito que me planteo con los diarios es reflexionar acerca de mi práctica docente e ir desarrollando una actitud crítica que me permita una mejora de la misma. En ellos llevo a cabo un proceso de retrospección, en el sentido de que se establecen relaciones entre mi historia de vida y mi historia profesional o, dicho de otra manera, entre mis vivencias como escolar y mis vivencias como docente" (Diario de clases. Marzo de 2005).

Llevar a cabo un proceso narrativo, desde este punto de vista, no sólo es una excelente herramienta de desarrollo profesional, sino también personal:

"Lo que procuro con el empleo de esta herramienta es ayudar a dar sentido a mi vida como profesional de la enseñanza y a los sucesos que me van aconteciendo a lo largo de la misma y que me van moldeando de una determinada manera" (Diario de clases. Febrero de 2005).

También es una manera de reflejar los miedos e inquietudes propias de los primeros años de profesión, tal y como él mismo relata:

"A veces me entra la duda de si podré ser docente de forma permanente. Son ya años de encierro voluntario, de privación de prácticamente todo, y no sé si compensa. Pero, si no me dedico a esto, ¿qué podré hacer?, ¿para qué sirvo?" (Diario del profesor. Febrero de 2010).

La decisión de continuar utilizando este recurso en el día a día del aula implica, para el educador, que los diarios cada vez vayan enriqueciendo más su práctica pedagógica, al tiempo que van dejando constancia de la evolución de su identidad profesional:

"Creo que los diarios cada vez son más ricos en información, se han convertido en una herramienta de carácter autoexpresivo. De las lecturas de los mismos extraigo la convicción de que, como educador, cada vez presto mayor atención a lo personal de los alumnos y a mis preocupaciones como docente y como persona y menos a lo curricular" (Diario de clases. Febrero de 2011).

Igual que sucedía con los relatos autobiográficos, los diarios de clase son otro de los instrumentos fundamentales para la recogida de datos que conforman su tesis doctoral. Gracias a ellos, cobran sentido algunas vivencias y situaciones de su práctica docente aunque, como él mismo explica, también en ocasiones son cuestionados:

"Hay gente que me pregunta cómo se puede investigar empleando un diario pues, innegablemente, los datos que de ahí emergen están impregnados de mi ideología. Pero el estudio no pretende proporcionar evidencias generalizables ni extensibles a otras situaciones sino, simplemente, servir de ayuda al que decida iniciarse en el mundo de la enseñanza mediante la reflexión de algunas cosas de las que hablo, la similitud contextual en la que se encuentra o sugiriendo lecturas que puedan resultarle significativas" (Historia de vida).

Asimismo, creemos conveniente resaltar que estos datos, igual que las autonarrativas, pueden llevar a un sentimiento de "fiscalización del propio trabajo", "una forma sutil de 
control sobre el profesor, en la medida en que se entra en la reconstrucción del espacio íntimo de su autobiografía" (Hernández, 2004, p. 13). Aun así, el educador es consciente de las ventajas de utilizar este recurso como modo de mejorar su práctica:

"En los diarios siempre presento las cosas tal y como han ocurrido y tal y como las he vivido. No pretendo aparentar que todo me sale bien. Todo lo contrario, a veces desvelan momentos $y$ situaciones en los que nada parece encajar" (Diario de clases. Mayo de 2010).

Nos mostramos convencidos de que, disponer de un diario de clases, permite profundizar en la docencia, detectar los puntos débiles de la actuación del profesor y, así, reorientar la enseñanza a partir de la observación y el análisis llevado a cabo.

Contar con esta herramienta, al igual que ocurre con las autonarrativas, permite al docente abrirse a un modelo de investigación "diferente", a un mayor desarrollo del pensamiento crítico y científico en torno a cuestiones pedagógicas que, a su vez, revierte en una mejor respuesta a la realidad que se encuentra en las aulas. Se posibilita, como vemos, modificar la actividad educativa en ciertos aspectos $\mathrm{y}$, asimismo, probar nuevas perspectivas curriculares surgidas de lecturas, pensamientos y reflexiones sobre los acontecimientos propios de la profesión.

El acercamiento de un docente a otros ámbitos de la investigación: los sentimientos propios de un "intruso"

Una vez terminados los estudios de maestro, nuestro docente comenzó a estudiar la licenciatura en Ciencias de la Actividad Física. En esta nueva etapa se abrieron ante él diferentes mundos por explorar, entre ellos, el de la investigación biomédica, no exentos de dificultades:

"Los meses que pasé en el Centro de Investigaciones Biológicas fueron complicados. Mi caso es particular, pues era el primer estudiante de Educación Física que iba a parar allí. ¿Qué podría aportar yo?, ¿acaso también los educadores físicos investigan? En estos meses fui más consciente de la concepción que se tiene de nuestra profesión" (Historia de vida).

Los sentimientos de intrusismo e incapacidad del educador le convencen del uso clasista que se hace de la ciencia y de la investigación y la presunción que se hace, de los profesionales de la Educación Física, de "expertos poco brillantes académicamente":

"Los investigadores de las diferentes áreas tienden a pensar que su campo de estudio es el más importante e imprescindible, y esto parece más evidente aún entre médicos y biólogos, profesionales con los que me codeaba a diario. Mientras que estos, en la hora del desayuno, departían acerca de curas del cáncer, los alimentos transgénicos y demás temas interesantes, a mí me hacían preguntas tan simples como si me gustaría formar parte de su equipo de fútbolsala para el partido anual del centro o el cuerpazo que debían tener mis compañeras de clase" (Historia de vida).

Paradójicamente, en el ámbito de las Ciencias Sociales la formación inicial del educador tampoco parecía tener cabida:

"Me puse en contacto con una investigadora del Instituto de Estudios Sociales que llevaba a cabo una línea de investigación sobre la discriminación racial del alumnado. Durante la charla me encontré con un obstáculo ya habitual: mi formación inicial no tenía cabida en esta línea de investigación. No deja de ser paradójico que, a pesar de que por expediente académico y por currículum parecía ser el más idóneo para optar a una de las becas predoctorales que ofertaban, desde el principio fui descartado por ser maestro titulado y estar cursando la licenciatura en Ciencias de la Actividad Física" (Historia de vida). 
La concepción que se tiene de la educación en general, y de la Educación Física en particular, está claramente influenciada socialmente. Esto es, si cabe, más evidente, si hablamos de investigación en estos campos. Afirmaciones tales como "en gimnasia no se investiga" o "no podrás si no tienes los conocimientos de un médico" van calando hondo en la manera de afrontar e intentar abrirse paso en el mundo de la investigación "más allá de lo permitido". Para el docente se hace necesario, entonces:

"Saber defender nuestra profesión como medio de hacer frente a las concepciones sociales, para crecer a través de la experiencia y para demostrar que la ciencia, ni es neutral, ni es patrimonio sólo de algunos afortunados" (Historia de vida).

Desde nuestra perspectiva, más que la formación inicial son la inquietud, la reflexión, la constancia y la determinación los pilares fundamentales de cualquier buen investigador; tener ese compromiso por cuestionarse sistemáticamente la propia manera de enseñar como base para el desarrollo es, entonces, fundamental para el educador que se responsabilice de estudiar y mejorar su profesión. $Y$ es que, aunque creemos que siempre existirán relaciones de poder en las que haya ciencias consideradas más importantes que otras, es importante defender y dar a conocer la importancia de investigar en educación a través de la creación de un clima de diálogo y entendimiento. Un diálogo capaz de mejorar la consideración de la educación y, al tiempo, capaz de ir rompiendo la distancia con personas de diferentes ideas, niveles y características que permitan el enriquecimiento mutuo.

\section{La participación en grupos de investigación: un intento por reforzar el estatus de la profesión}

La ya mencionada baja consideración social de nuestra profesión puede paliarse, en parte, colaborando en grupos de investigación. El paso por la Enseñanza Secundaria demuestra a nuestro docente que compartir las aportaciones que la Educación Física puede hacer al mundo de la ciencia ayuda a superar la concepción que se tiene sobre la materia:

"Un compañero se ha enterado de que voy a trabajar como asociado en la universidad y me ha preguntado irónicamente que cómo era posible, que él tiene el título de doctor, publicaciones y no ha conseguido trabajar en la universidad. Le he hablado de algunas de mis publicaciones o de mis acercamientos a la investigación; se ha sorprendido de que fuera así, mostrando un interés que espero que fuera sincero y adoptando un tono más cordial. No sé si la sorpresa inicial se la llevó tanto por ser yo quien soy o por ser profesor de lo que soy" (Diario del profesor. Febrero de 2011).

Son estas publicaciones sobre diferentes temáticas de las Ciencias de la Actividad Física y de la Educación Física las que sirven de aval y soporte a su credibilidad como docente y a la profesión de educador físico, más aún durante la etapa de Enseñanza Secundaria, en la que comparte centro con varias personas con el título de doctor y con dilatada experiencia en el mundo investigadora:

"He llevado algunos de los artículos que me han publicado para que los pudieran leer dos compañeros. Los dos son del ámbito biomédico y están publicados en revistas de impacto lo que, según parece, les da algo más de "credibilidad". Me han preguntado cómo he llegado a documentarme y a trabajar en esos proyectos de investigación, y les he estado hablando de cómo me introduje en estos temas. me gusta la sensación de compartir mis logros con otros profesionales de la educación, a ver si así se va cambiando la idea que se tiene del profesor de Educación Física" (Diario del profesor. Mayo de 2011).

Las dificultades a las que he de hacer frente como especialista en Educación Física a la hora de querer llevar a cabo tareas de investigación no merman el afán por querer comprender, 
analizar y enfrentarse a diferentes situaciones de mi práctica. Por el contrario, la participación del docente en proyectos de investigación le reporta beneficios tanto a él como a su alumnado:

"Gracias a mi participación en proyectos y grupos de investigación me voy modelando como un profesor con inquietudes académicas, con un mayor conocimiento de la asignatura y con ilusión por seguir aprendiendo y mejorando, lo que me permite suplir mi falta de experiencia con una mayor pericia y seguridad" (Diario del profesor. Abril de 2011).

En este sentido, los educadores de cualquier nivel educativo en general, y los docentes de Educación Física en particular, hemos de involucrarnos en procesos de investigación educativa no sólo como medio de mejora de la práctica docente sino, también, como modo de ganar prestigio profesional a través de la creación de un conocimiento compartido con el resto de la comunidad educativa. Sin embargo, el tiempo que nuestro docente pasa ejerciendo como profesor asociado, le convencen de la distancia que separa el mundo académico universitario del mundo de las aulas:

"Me doy cuenta de lo diferentes que son las exigencias en la universidad y en el instituto. En el instituto a pocos se les ocurre pararse a pensar que haya que investigar con el objeto de mejorar la docencia. Además, las publicaciones sobre aspectos pedagógicos en ocasiones son tan complicadas y académicas que no se ajustan a la realidad de las aulas, lo que lleva a un distanciamiento entre el conocimiento científico y nuestro día a día. Es una pena que no existan unas relaciones más estrechas entre el ámbito universitario y el no universitario, que el profesorado de uno esté tan lejano del otro" (Diario del profesor. Marzo de 2011).

En cualquier caso, buscar ese acercamiento es un esfuerzo que merece la pena pues, como explica el docente:

"Formar parte de proyectos que tienen una aplicación directa en el aula me ayudan a fomentar mis habilidades de enseñanza y obtener nuevas ideas para poner en marcha en mi práctica docente. Suponen una mejora significativa en la calidad de mi enseñanza" (Diario del profesor. Noviembre de 2012).

Más aún, y como él mismo explica, suponen una evolución en su identidad profesional, "pues ahora no busco tanto mejorar el prestigio social de la profesión docente por medio de la participación en programas de investigación, sino que ahora considero más relevante mejorar mi enseñanza". Sobre este particular, nos mostramos convencidos de que es responsabilidad de cada educador no sólo investigar y reflexionar sobre su propia práctica sino, más aún, aportar lo que esté en su mano de manera que redunde en la mejora del proceso de enseñanza/aprendizaje para el conjunto de la comunidad educativa.

Por otra parte, esta manera de investigar tiene un claro componente individual que transforma el lenguaje, las actividades y las prácticas docentes, pero su potencial se ve empobrecido si no se acompaña de un componente grupal que ayude a transformar los discursos, las prácticas sociales y la organización social (Kemmis, 1999). En este sentido, nuestro educador va evolucionando y participando en grupos de investigación-acción que permiten mejorar su práctica y abrir su conocimiento a diferentes perspectivas:

"Es una suerte poder formar parte de proyectos de innovación docente en los que comparto tiempo, experiencias y aprendizajes con otros profesionales de la enseñanza. Hasta ahora, casi todo lo que he hecho en el ámbito de la educación lo he ido haciendo yo solo, e incluso en muchas ocasiones he tenido sentimientos de verdadera soledad. [,...]. Ahora formo parte de dos proyectos en los que, a través de un diálogo entre iguales, vamos intercambiando conocimientos, resultados, aprendiendo unos de otros, mejorando el modo de afrontar la profesión..." (Diario del profesor. Diciembre de 2012). 
Comprobamos, así, cómo la identidad profesional e investigadora del educador se encuentran en continuo proceso de revisión y evolución. Es un proceso inacabado, en el que el educador comienza desde una perspectiva meramente individual para, paulatinamente, ir rompiendo el tan frecuente individualismo que subyace en la labor docente a otra forma de trabajo que implique relaciones sociales, colaboración y diálogo con otros compañeros y profesionales (Kemmis, 1993, 1999; Pollard \& Tann, 1997).

\section{Discusión}

Gracias a los relatos que se han ido presentando a lo largo del texto, el lector se ha podido hacer una idea del camino investigador que ha seguido un docente novel y lo que para él y para su profesión ha supuesto. Por otra parte, hemos intentado ofrecer una perspectiva diferente sobre la investigación y el modo de llevarla a cabo, entendiendo que los relatos autobiográficos aquí presentados no tienen un trasfondo de confesión sino que, en la línea que propone Richardson (1994, en Armour, 2004, p. 127), "escribo porque quiero descubrir algo. Escribo para aprender algo que no sabía antes de escribirlo".

En este sentido, una de las conclusiones a las que debería llegar el lector es que, investigar a partir de los diarios de clase y los relatos autobiográficos, ayuda a plasmar muchas de las ideas y experiencias que van configurando la identidad profesional del educador. En este sentido, puede ser interesante fomentar no sólo en la comunidad científica, sino en la comunidad educativa en general, la realización de estudios que permitan sacar a la luz la dimensión personal del docente que sirvan para invitar a otros profesionales a reflexionar y compartir conocimiento de manera honrada, transformadora y sugerente. Haciéndolo así, estaremos modelando educadores con inquietudes académicas, con mayor conocimiento de las asignaturas que imparten, con ilusión por seguir aprendiendo y mejorando (lo que, en el caso de educadores inexpertos como el que aquí hemos presentado, suple su falta de experiencia con una mayor pericia y seguridad) y, también, con sensibilidad educativa, siendo éstas las características que definen al docente preocupado por mejorar su práctica con/para el alumnado.

Para hacerlo posible, es necesario imbricar docencia e investigación pues, como hemos apuntado, esta última parece estar reservada a ciertos sectores. En lo que a nosotros nos interesa, es habitual que aquellos que desempeñan su labor en las etapas de Educación Infantil, Primaria y Secundaria desconozcan los resultados de las investigaciones que se llevan a cabo en las instituciones académicas, y no es habitual que se comprometan en dinámicas investigadoras (Gimeno, 1999; Jackson, 2010; Rockwell, 2009). Sin embargo, existe cierta tendencia a creer que las reformas educativas se pueden mejorar y hacer más efectivas si se involucra a los educadores en programas de investigación e innovación educativa. Estos programas de investigación permitirán a los docentes profundizar en temas de su interés que redunden en una mejora de su labor profesional y, así, incorporar los resultados de los mismos a su práctica docente. Participar en proyectos de investigación, desde esta perspectiva, ayuda al profesorado a involucrarse, explorar y conocer teorías y conceptos y las implicaciones que éstos tienen en su campo (Henson, 2001). Espolear una actitud investigadora entre el profesorado implica, entonces, romper con la creencia de que la investigación del profesorado no garantiza un conocimiento objetivo (Elliott, 2010), suprimiendo "la paradoja de los dos mundos (el académico y el real). Es como la visión paternalista del amo y la esclavitud de los obreros" (Imbernón, 2002, p. 8). Si, por el contrario, continuamos considerando que la elaboración y producción del conocimiento teórico, técnico y experto es patrimonio de la investigación universitaria y que el maestro no necesita acceder al conocimiento científico, sino únicamente a los 
conocimientos elaborados por los expertos, estaremos limitando la formación reflexiva (y, por ende, investigadora) del profesorado (Arnaus, 1999; Medina, Jarauta, \& Imbernón, 2010).

Como hemos explicado, la participación del profesor en proyectos de investigación no reporta beneficios únicamente al educador, sino que los estudiantes también pueden verse favorecidos por la misma (Jove, 2011), al ofrecerles la oportunidad de aprender más allá de los conocimientos del docente y al ser éste más capaz de transferir al aula sus experiencias y observaciones llevadas a cabo durante los programas de investigación (Burnaford, Fischer, \& Hobson, 2001).

De ahí que creamos que, desde la formación inicial, es necesario ayudar a los (futuros) educadores a involucrarse en proyectos de investigación como medio de mejorar y enriquecer nuestra práctica, como modo de alentar sus aptitudes y actitudes reflexivas y, también, alentar su espíritu colaborativo en pro de crear un conocimiento compartido con el resto de la comunidad educativa. Haciéndolo así, estaremos rompiendo con esa opresión a que a menudo nos somete el uso clasista de la ciencia para dar paso a una relación armónica entre las diferentes disciplinas, en las que no tengan cabida los etiquetajes que algunos se empeñan en poner y ofreciendo un clima de desarrollo y de transformación personal y profesional.

Es decir, investigar sobre la propia práctica requiere de cierta fuerza de voluntad por parte del educador y, sobre todo, una profunda convicción de su pertinencia si se pretende romper con el pensamiento relacionado con la "desprofesionalización y las resistencias a la formación” (Imbernón, 2007, p. 144) tan habituales en nuestra labor. Quizá, para ello, debamos recordar, como explica Marina (1995), que en el ámbito de la educación todos braceamos el mismo mar, en el que nadie es más o menos importante que los demás, y donde es posible compartir intereses y pasiones sobre un tema, compartir experiencias y fortalecer las relaciones.

\section{A modo de conclusión: ¿qué aporta (re)pensarse como educador investigador en el aula?}

Nuestra pretensión en este escrito no ha sido otra que mostrar el camino por el cual un educador inexperto va descubriendo la importancia de investigar acerca de su práctica educativa. Tal y como hemos explicado, en ocasiones no es un camino fácil ni cuenta con el beneplácito de otros profesionales (pertenezcan, o no, al mundo de la enseñanza). Pero no queremos terminar ofreciendo una imagen pesimista acerca de esta "otra" manera de investigar. Al contrario: estamos convencidos de que, cambiar la mirada hacia esta alternativa investigadora, permite dar un paso adelante en la profesionalización docente, enriqueciendo y mejorando la escuela, la comunidad educativa y, en general, la sociedad a la que, como educadores, nos debemos. Desde luego que los relatos de los educadores no conforman un todo, y no es eso lo que aquí hemos pretendido reflejar. Simplemente queremos destacar que la voz de los educadores, a través de las historias que ellos nos cuentan, pueden resultar útil en sí misma si se le presta la debida atención.

Por otro lado, hemos defendido la necesidad de que sean los maestros y profesores quienes lleven a cabo una investigación sistemática de su propia práctica, rompiendo así con la escasa correlación entre el mundo de la investigación educativa que parte del profesorado universitario y el mundo real de las aulas o, si se prefiere, entre el mundo de los académicos y el de los prácticos. Si se favorece la implicación de los educadores en procesos de investigación de su práctica a partir de estrategias como las que aquí hemos 
presentado (diario de clases y narrativas autobiográficas, fundamentalmente), creemos que puede estimularse la comprensión y transformación de su enseñanza, de manera que puedan estructurar su docencia de una manera crítica y razonada (Erickson, 1990). Cierto es que se trata de un tipo de investigación en primera persona, con un claro carácter individual, pero imbuida de un claro carácter de transformación social: si uno aprende a ser mejor docente, será capaz de mejorar la educación que imparte a sus estudiantes y enriquecerá y mejorará la concepción actual que, desde determinados sectores, se tiene de la labor educadora.

\section{Bibliografía}

Álvarez, C. (2008). La etnografía como modelo de investigación en educación. Gazeta de Antropología, 24(1),

Armour, K. M. (2004). "Lugares enclavados": reflexiones sobre mis entrevistas al profesorado de Educación Física. En A. Sicilia \& J. M. Fernández-Balboa (Eds.), La otra cara de la investigación. Reflexiones desde la Educación Física (pp. 127-137). Sevilla: Wanceulen.

Arnaus, P. (1999). La formación del profesorado: un encuentro comprometido con la realidad educativa. En A. Pérez, J. Barquín \& J. F. Angulo (Eds.), Desarrollo profesional del docente. Política, investigación y práctica (pp. 599-635). Madrid: Akal.

Barba, J. J. (2001). Aprendiendo a hacer etnografía durante el Prácticum. Revista Interuniversitaria de Formación del Profesorado, 42, 177-190.

Bolívar, A., Domingo, J., \& Fernández, M. (1998). La investigación biográfico-narrativa en educación. Guía para indagar en el campo. Granada: Universidad de Granada.

Bolívar, A., Domingo, J., \& Fernández, M. (2001). La investigación biográfico-narrativa en educación. Enfoque y metodología. Madrid: La Muralla.

Buendía, L. (2010). Metodologías de investigación para el desarrollo del conocimiento en un contexto multicultural. En A. Boza, J. M. Méndez, M. Monescillo \& M. Toscano (Eds.), Educación, investigación y desarrollo social (pp. 15-30). Madrid: Narcea.

Burnaford, G. E., Fischer, J., \& Hobson, D. (2001). Teachers doing research: The power of action through inquiry. London: Lawrence Erlbaum.

Chacón, M., \& Chacón, A. (2006). Los diarios de prácticas: una estrategia de reflexión en la formación docente. Acción Pedagógica, 15, 120-127.

Delgado, J. M., \& Gutiérrez, J. (1999). Métodos y técnicas cualitativas de investigación en Ciencias Sociales. Madrid: Síntesis.

Devís, J., \& Sparkes, A. (2004). La crisis de identidad de un estudiante universitario de Educación Física: la reconstrucción de un estudio biográfico. En A. Sicilia \& J. M. Fernández-Balboa (Eds.), La otra cara de la investigación. Reflexiones desde la Educación Física (pp. 83-106). Sevilla: Wanceulen.

Devís, J., \& Sparkes, A. C. (1999). Burning the book: A biographical study of a pedagogically inspired identity crisis in Physical Education. European Physical Education Review, 5(2), 135-152.

Elliott, J. (2010). El "estudio de la enseñanza y el aprendizaje": una forma globalizadora de investigación del profesorado. Revista Interuniversitaria de Formación del Profesorado, 24(2), 223-242. 
Erickson, F. (1990). Métodos cualitativos de investigación sobre la enseñanza. In M. C. Wittrock (Ed.), La investigación de la enseñanza (Vol. II, pp. 195-301). Barcelona: Paidós.

Feliu, J. (2007). Nuevas formas literarias para las Ciencias Sociales: el caso de la autoetnografía. Athenea Digital, 12, 262-271

Fernández, M. (1985). Cualquier día a cualquier hora: invitación a una etnografía de la escuela. Arbor, 477, 57-87.

Gimeno, J. (1999). Poderes inestables en educación Madrid: Morata.

Goetz, J. P., \& LeCompte, M. D. (1988). Etnografía y diseño cualitativo en investigación educativa. Madrid: Morata.

González, G. (2013). Evolución de la identidad profesional de un docente novel de Educación Física: análisis a partir de la reflexión de sus experiencias personales y de su propia práctica. Tesis Doctoral. Universidad de Valladolid, Valladolid.

González, G., \& Martínez, L. (2009). Aproximación a los significados e interpretaciones de la lesión en futuros docentes de Educación Física por medio de narraciones autobiográficas. Retos: Nuevas Tendencias en Educación Física, Deporte y Recreación, 15(46), 35-40.

González, G., Barbero, J. I., Bores, N. J., \& Martínez, L. (2013). Relatos autobiográficos sobre la incompetencia motriz aprendida de un docente novel de EF y sus posibilidades autoformativas. Revista Iberoamericana de Educación, 62, 39-56.

González, G., Barba, J. J. (en prensa). La perspectiva autobiográfica de un docente novel sobre los aprendizajes de Educación Física en diferentes niveles educativos. Cultura, Ciencia y Deporte.

Hammersley, M., \& Atkinson, P. (1994). Etnografía. Métodos de investigación. Barcelona: Paidós.

Henson, R. K. (2001). The effects of participation in teacher research on teacher efficacy. Teaching and Teacher Education, 17(7), 819-836.

Hernández, F. (2004). Las historias de vida como estrategia de visibilización y generación de saber pedagógico. In I. Goodson (Ed.), Historias de vida del profesorado (pp. 9-26). Barcelona: Octaedro.

Imbernón, F. (2002). La investigación educativa como herramienta de formación del profesorado. Reflexión y experiencias de investigación educativa. Barcelona: Graó.

Imbernón, F. (2007). La formación y el desarrollo profesional del profesorado. Hacia una nueva cultura profesional. Barcelona: Graó.

Jackson, P. W. (2010). La vida en las aulas. Madrid: Morata.

Jove, G. (2011). How do I improve what I am doing as a teacher, teacher educator and action-researcher through reflection? A learning walk from Lleida to Winchester and back again. Educational Action Research, 19(3), 261-278.

Kemmis, S. (1993). El curriculum: más allá de la teoría de la reproducción. Madrid: Morata.

Kemmis, S. (1999). La investigación-acción y la política de la reflexión. En A. Pérez, J. Barquín \& J. F. Angulo (Eds.), Desarrollo profesional del docente. Política, investigación y práctica (pp. 95-118). Madrid: Akal. 
Latorre Beltrán, A. (1992). La reflexión en la formación del profesor. Tesis Doctoral. Universitat de Barcelona, Barcelona.

Liston, D. P., \& Zeichner, K. M. (2003). Formación del profesorado y condiciones sociales de la escolarización. Madrid: Morata.

López, M. (2002). Las narrativas de los docentes en ambientes culturalmente diversos como vía de aproximación a sus teorías. Un estudio de caso. Universitas Tarraconensis. Revista de Ciències de l'Educació, 26, 9-28.

Marina, J. A. (1995). Ética para náufragos. Barcelona: Anagrama.

Martínez, J. (2008). El olvido de la investigación-acción en el asesoramiento docente y la innovación educativa. Profesorado: Revista de currículum y formación del profesorado, 12(1), 1-10.

Medina, J. L., Jarauta, B., \& Imbernón, F. (2010). La enseñanza reflexiva en la educación superior. Barcelona: Octaedro.

Ortega, F., \& Velasco, A. (1991). La profesión de maestro: un estudio sociológico del magisterio de Castilla la Mancha. Madrid: CIDE.

Pallma, S., \& Sinisi, L. (2004). Tras las huellas de la etnografía educativa. Cuadernos de Antropología Social, 19, 121-138.

Pollard, A., \& Tann, S. (1997). Reflective teaching in the Primary School: A handbook for the classroom. London: Cassell.

Rockwell, E. (2009). La experiencia etnográfica. Historia y cultura en los procesos educativos. Buenos Aires: Paidós.

Silvennoinen, M. (1994). ¡Todos en fila! La experiencia de la Educación Física Escolar y la imagen del papel del profesor. En J. I. Barbero (Ed.), Investigación alternativa en Educación Física: II Encuentro Unisport sobre Sociología Deportiva (pp. 271-284). Málaga: Unisport.

Silvennoinen, M. (2001). Relatos sobre deporte e identidad en mujeres y hombres. En J. Devís (Ed.), La Educación Física, el deporte y la salud en el Siglo XXI (pp. 203-212). Alicante: Marfil.

Smyth, J. W. (1991). Una pedagogía crítica de la práctica en el aula. Revista de Educación, 294, 275-300.

Sparkes, A. (1994). Life histories reflection and Physical Education teacher education: Exploring the possibilities. En L. Montero \& J. M. Vez (Eds.), Las didácticas específicas en la formación del profesorado (Vol. I, pp. 555-567). Santiago de Compostela: Tórculo.

Sparkes, A. (2001). Las identidades deportivas y el cuerpo: una relación problemática. En J. Devís (Ed.), La Educación Física, el deporte y la salud en el siglo XXI (pp. 181-201). Alcoy: Marfil.

Sparkes, A. (2004). La narración del cuerpo en la Educación Física y el deporte. En A. Sicilia \& J. M. Fernández-Balboa (Eds.), La otra cara de la investigación. Reflexiones desde la Educación Física (pp. 49-58). Sevilla: Wanceulen.

Surbeck, E., Han, E., \& Moyer, J. (1991). Assessing reflective responses in journals. Educational Leadership, 48(6), 25-27. 
Taylor, S. J., \& Bogdan, R. (1998). Introducción a los métodos cualitativos de investigación: la búsqueda de significados. Barcelona: Paidós.

Velasco, H. M., \& Díaz, A. (2004). La lógica de la investigación etnográfica: un modelo de trabajo para etnógrafos de la escuela. Madrid: Trotta.

Woods, P. (1998). Investigar el arte de la enseñanza: el uso de la etnografía en la educación. Barcelona: Paidós.

Zabalza, M. A. (2008). Diarios de clase: un instrumento de investigación y desarrollo profesional. Madrid: Narcea.

Zeichner, K. M., \& Liston, D. P. (1999). Enseñar a reflexionar a los futuros docentes. En A. Pérez, J. Barquín \& J. F. Angulo (Eds.), Desarrollo profesional del docente. Política, investigación y práctica (pp. 506-532). Madrid: Akal. 


\section{Autores}

Gustavo González Calvo

Calle De Las Mercedes número 8, $7^{\circ}$ D. 47006 Valladolid

Teléfono: 983-222782 Teléfono móvil: 647-545915

Dirección electrónica: gustavogonzalezcalvo@gmail.com

Doctor en Ciencias de la Educación por la Universidad de Valladolid, Licenciado en Ciencias de la Actividad Física y del Deporte y Graduado en Educación Física y Educación Primaria. Funcionario de Carrera del Cuerpo de Maestros y profesor-colaborador del Departamento de Expresión Corporal de la Universidad de Valladolid.

Participa en proyectos de innovación docente como el Grupo ACOGE (Educación, Inclusión e Igualdad) y el proyecto MultiScopic (desarrollo de comunidades de práctica internacionales en la formación inicial y permanente del profesorado de Educación Primaria). Sus líneas de investigación se centran en el desarrollo de la identidad reflexiva del profesorado, la investigación a través de historias de vida y las etnografías escolares

Raquel Becerril González

Plaza de España número 3. 34220 Magaz de Pisuerga (Palencia)

Teléfono: 661709492

Dirección electrónica: raquelbz@pdg.uva.es

Diplomada en Educación Social y Licenciada en Pedagogía, con la especialidad de Pedagogía Social y Laboral. Trabaja como educadora social en el Ayuntamiento de Magaz de Pisuerga y es profesora-colaboradora del Departamento de Pedagogía de la Universidad de Valladolid.

Forma parte del grupo de investigación ICUFOP (Investigación del currículum y formación del profesorado), y realiza su tesis doctoral en el área de Educación Física 